\title{
Association between electrocardiographic findings and cardiac dysfunction in adult isolated traumatic brain injury
}

\author{
Vijay Krishnamoorthy ${ }^{1}$, Sumidtra Prathep ${ }^{1}$, Deepak Sharma ${ }^{1,2}$, Edward Gibbons ${ }^{3}$, \\ Monica S. Vavilala ${ }^{1,2,4,5}$
}

Introduction: Abnormal electrocardiographic (ECG) findings can be seen in traumatic brain injury (TBI) patients. ECG may be an inexpensive tool to identify patients at high risk for developing cardiac dysfunction afterTBI.The aim of this study was to examine abnormal ECG findings after isolated TBI and their association with true cardiac dysfunction, based on echocardiogram. Methods: Data from adult patients with isolated TBI between 2003 and 2010 was retrospectively examined. Inclusion criteria included the presence of a 12 -lead ECG within $24 \mathrm{~h}$ of admission and a formal echocardiographic examination within $72 \mathrm{~h}$ of admission after TBI. Patients with preexisting cardiac disease were excluded. Baseline clinical characteristics, I 2-lead ECG, and echocardiogram report were abstracted. Logistic regression was used to identify the relationship of specific ECG abnormalities with cardiac dysfunction. Results: We examined data from 59 patients with isolated TBI who underwent I2-lead ECG and echocardiographic evaluation. In this cohort, I 3 (22\%) patients had tachycardia (heart rate > $100 \mathrm{bpm}$ ), 25 (42.4\%) patients had a prolonged QTc, and $6(10.2 \%)$ patients had morphologic end-repolarization abnormalities (MERA), with each having an association with abnormal echocardiographic findings: Odds ratios (and 95\% confidence intervals) were 4.14 (1.02-17.05), 9.0 (I.74-46.65), and 5.63 (1.96-32.94), respectively. Ischemic-like ECG changes were not associated with echocardiographic abnormalities. Conclusions: Repolarization abnormalities (prolonged QTc and MERA), but not ischemic-like ECG changes, are associated with cardiac dysfunction after isolated TBI. I2-lead ECG may be an inexpensive screening tool to evaluate isolated TBI patients for cardiac dysfunction prior to more expensive or invasive studies.

Keywords: Cardiac function, electrocardiography, traumatic brain injury

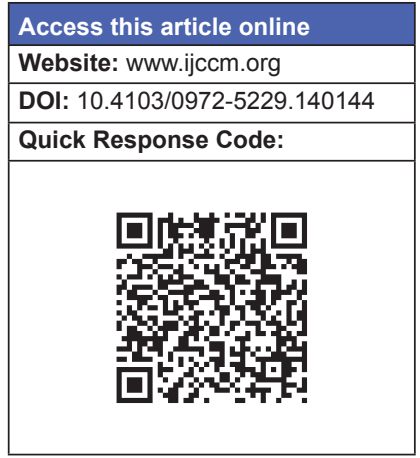

\section{Introduction}

Traumatic brain injury (TBI) is a major public health concern and a leading cause of traumatic death worldwide. ${ }^{[1]}$ Complications after TBI known as second insults can worsen neurologic and patient outcomes. ${ }^{[2,3]}$ Clinically, post-TBI hypotension (systolic blood pressure $<90 \mathrm{mmHg}$ ) has been directly linked to

From:

Departments of ${ }^{1}$ Anesthesiology and Pain Medicine, ${ }^{2}$ Neurological Surgery, ${ }^{3}$ Cardiology, ${ }^{4}$ Pediatrics and ${ }^{5}$ Radiology, University of Washington, Seattle, WA, USA

\section{Correspondence:}

Dr. Vijay Krishnamoorthy, Department of Anesthesiology and Pain Medicine, University of Washington, Harborview Medical Center, 325 Ninth Avenue,

P. O. Box 359724, Seattle, WA 98104, USA. E-mail: vkrish@uw.edu mortality and poor disposition. ${ }^{[4,5]}$ While the majority of patients experience a hypotensive episode during TBI surgery, ${ }^{[6]}$ the cause is often unknown, and treatment may be empiric. Cardiac dysfunction has been documented in TBI, and implicated as a cause for hypotension in other brain injury paradigms, where experimental evidence postulates that brain-heart-lung interactions may cause myocardial dysfunction in TBI. ${ }^{[7,8]}$ This suggests that the cardiac dysfunction may be one reason for post-TBI hypotension.

Abnormal electrocardiographic (ECG) findings can also be associated with neurologic hemorrhage, where both ischemic-like changes and a variety of repolarization 
abnormalities have been described..$^{[9,10]}$ Autonomic instability has been described in small series in adults and children with TBI, with noted changes in heart rate variability thought to be secondary to sympathetic over-activity and autonomic imbalance. ${ }^{[11,12]}$ In addition to repolarization abnormalities and ischemic-like ECG changes, heart rate variability may be blunted in brain-injured patients, reflecting dysfunction in the autonomic nervous system. ${ }^{[11,12]}$ Early ECG may serve as an inexpensive test to screen for cardiac dysfunction prior to ordering more expensive and potentially more invasive testing. The aim of this study was to examine abnormal ECG findings early after isolated TBI and their association with cardiac dysfunction.

\section{Methods}

\section{Setting}

This retrospective study was performed at Level 1 trauma center with separate Intensive Care Units (ICUs) for medical, trauma/surgical, burn, and neurology/ neurosurgery patients. All ICUs have 24-h in-house physician coverage and are staffed by intensivist-led teams consisting of an attending physician, critical care fellow, senior level, and junior level resident. The Departments of Surgery, Anesthesiology, and Internal Medicine provide physician coverage.

\section{Patients}

After Institutional Review Board approval, data were abstracted from our institution's trauma registry, which includes complete admission and hospitalization records for all patients with traumatic injury that were triaged to our Level 1 trauma center. We identified all adult (age $>18$ years) patients with isolated TBI who received care between 2003 and 2010. Inclusion criteria included the presence of a 12-lead ECG within $24 \mathrm{~h}$ of admission and a formal echocardiogram within $72 \mathrm{~h}$ of admission after TBI. Patients with preexisting cardiac disease were excluded. Baseline clinical characteristics, 12-lead ECG, and echocardiogram report were abstracted. ECG tracings were interpreted by a blinded cardiologist.

\section{Clinical care}

Traumatic brain injury was categorized as mild (Glasgow Coma Scale [GCS] 13-15), moderate (GCS 9-12) and severe (GCS 3-8); TBI was also categorized by head Abbreviated Injury Scale: 1 = Minor, 2 = Moderate, $3=$ Serious, $4=$ Severe, $5=$ Critical, and $6=$ Not survivable, ${ }^{[13]}$ as previously described. During the study period, patients were resuscitated according to institutional practice, consistent with the Brain Trauma Foundation guidelines. ${ }^{[14,15]}$ Relevant to this study, practice included invasive intracranial pressure monitoring, maintaining intracranial pressure $<20 \mathrm{mmHg}$, minimum cerebral perfusion pressure (CPP) of $50 \mathrm{mmHg}$, $\mathrm{PaCO}_{2} 35-40 \mathrm{mmHg}, \mathrm{SaO}_{2}>90 \%$ and maintaining core body temperature between $35^{\circ} \mathrm{C}$ and $37.5^{\circ} \mathrm{C}$ with antipyretics, cooling/warming blankets, or intravascular cooling devices if needed. Practices involving requests for echocardiography were not standardized during the time of this study, and echocardiography was requested at the discretion of the primary attending intensivist.

\section{Statistical analyses}

Statistical analyses were performed using SPSS 19.0 (IBM, SPSS, Inc., Chicago, Illinois, USA). Descriptive statistics was used to describe clinical characteristics, ECG findings, echocardiographic findings, head computed tomography findings and in-hospital mortality. Data are presented as mean and standard deviation for parametric data, median and standard error of the mean for nonparametric data, or as percentages for categorical variables. Student's t-test was used to examine differences between these normally distributed continuous variables. Mann-Whitney U-test was used to test for differences in these nonparametric continuous data. Chi-square test was used to test for differences between categorical variables.

Univariate analysis was used to examine the relationship between abnormal ECG findings (tachycardia [heart rate $>100 / \mathrm{min}]$, nonsinus rhythm, prolonged PR interval [PR $>0.20 \mathrm{~s}$ ], prolong QRS interval [QRS $>1.2 \mathrm{~s}$ ], prolonged QTc duration [QTc $>440 \mathrm{~ms}$ in male, QTc $>470$ $\mathrm{ms}$ in female], left ventricular hypertrophy, ischemic-like patterns, and morphological end-repolarization abnormalities [MERA]) and abnormal echocardiography. Cardiac dysfunction was defined as ejection fraction $<50 \%$ or the presence of regional wall motion abnormality (RWMA) on surface echocardiogram.

\section{Results}

\section{Clinical characteristics}

We examined data from 59 patients with isolated TBI whose medical records contained an ECG within $24 \mathrm{~h}$ of admission and at least one echocardiography report within $72 \mathrm{~h}$ of admission. Baseline clinical characteristics are shown in Table 1. Patients were between 18 and 89 (median $63 \pm 2$ ) years and $61 \%$ were male. Patients had a median GCS score at admission of $10 \pm 5$, and a median baseline injury severity score of $20 \pm 1$. The majority of patients had a subdural hematoma (59.3\%), followed by subarachnoid hemorrhage (SAH) (13.6\%), intraparenchymal hemorrhage $(11.9 \%)$, cerebral 
contusions (10.1\%), and other lesions (5.1\%). Overall all cause in-hospital mortality in this patient sample was $11.9 \%$.

\section{Electrocardiographic and echocardiographic and findings}

Electrocardiographic and echocardiographic findings are shown in Table 2. The median heart rate was $79 \pm 3$ bpm, with approximately $80 \%$ being in a sinus rhythm. Prolonged PR interval occurred in $6.8 \%$ of patients, while a prolonged QTc was diagnosed in $42.4 \%$ of patients. Ischemic-like ECG changes (ST-depression, ST-elevation, Q-wave) occurred in a small number of patients (3.4\%, $3.4 \%, 6.8 \%$ respectively). Inverted T-waves occurred in $11.9 \%$ of patients, and MERA were observed in $10.2 \%$ of patients. Overall, $15.3 \%$ had a reduced left-ventricular ejection fraction, and $13.6 \%$ had a documented RWMA.

At the time of ECG, 13 (22\%) patients had tachycardia, $25(42.4 \%)$ patients had a prolonged QTc, and $6(10.2 \%)$ patients had MERA, with each having a univariate association with cardiac dysfunction as shown in Table 3: 4.17 ([1.02-17.05]; $P=0.04$ ), 9.0 ([1.74-46.65]; $P=0.003$ ), and 5.63 ([1.96-32.94]; $P=0.03$ ), respectively. Early ischemic-like ECG changes were not associated with echocardiographic abnormalities.

\section{Discussion}

The two main findings of our study were that ECG changes are common after TBI, and specific changes, particularly repolarization abnormalities, were associated with cardiac dysfunction based on echocardiography. These findings may help identify patients who may develop cardiac dysfunction based on an inexpensive test prior to ordering further studies; in addition, knowledge of cardiac

Table I: Clinical characteristics of 59 patients with isolated TBI

\begin{tabular}{lc}
\hline Clinical characteristics & \\
\hline Age (years) & $63 \pm 2(I 8-89)$ \\
Male gender (\%) & 6 I \\
Admission GCS score (\%) & $10 \pm 5$ \\
Mild TBI (GCS I3-I5) & $23(39.0)$ \\
Moderate TBI (GCS 9-I2) & $5(8.5)$ \\
Severe TBI (GCS <8) & $31(52.5)$ \\
Head AIS 4-6 & $46(78.0)$ \\
ISS* & $20 \pm$ I \\
Computed tomography diagnosis (\%) & \\
Subdural hematoma & $35(59.3)$ \\
Subarachnoid hemorrhage & $8(13.6)$ \\
Intraparenchymal hemorrhage & $7(I I .9)$ \\
Contusion & $6(I 0.1)$ \\
Other & $3(5.1)$ \\
Midline shift & $14(23.7)$ \\
All cause in-hospital mortality & $7(I I .9)$ \\
\hline Data as $n$ (\%) or median \pm SEM. TBI: Traumatic brain injury; SEM: Standard error of the \\
mean; GCS: Glasgow coma scale; AlS: Abbreviated injury score; ISS: Injury severity score
\end{tabular}

dysfunction may allow a more rational use of vasopressors and possibly the institution of cardioprotective measures, as nonneurologic organ dysfunction after TBI is associated with worsened mortality. ${ }^{[16]}$

Electrocardiographic changes are not uncommon after neurologic hemorrhage, with data to suggest that they may have an influence on patient outcome; for example, in aneurysmal SAH, ischemic like changes conferred a higher mortality ${ }^{[17]}$ Early repolarization abnormalities and ischemic-like ECG changes are common in both $\mathrm{SAH}$ and intracerebral hemorrhage ${ }^{[10]}$ The magnitude of ECG changes seems to correlate with severity of the

Table 2: ECG and echocardiographic findings in 59 patients with isolated TBI

\begin{tabular}{|c|c|}
\hline HR (bpm) & $79 \pm 3(46-158)$ \\
\hline Sinus rhythm, $n(\%)$ & $47(79.7)$ \\
\hline Arrhythmia, $n(\%)$ & $12(20.3)$ \\
\hline Prolong PR interval, $n$ (\%) & $4(6.8)$ \\
\hline PR interval duration (median \pm SEM (ms)) & $156 \pm 4(112-270)$ \\
\hline Prolonged QRS, n (\%) & $7(I 1.9)$ \\
\hline QRS (median \pm SEM (ms)) & $90 \pm 2(72-166)$ \\
\hline Prolonged QTc, n (\%) & $25(42.4)$ \\
\hline QTc (median \pm SEM (ms)) & $446 \pm 5(382-610)$ \\
\hline LVH, $n(\%)$ & $6(10.2)$ \\
\hline ST depression, $n$ (\%) & $2(3.4)$ \\
\hline ST elevation, $n(\%)$ & $2(3.4)$ \\
\hline Q wave, $n(\%)$ & $4(6.8)$ \\
\hline Inverted T wave, $n$ (\%) & $7(11.9)$ \\
\hline MERA, $n$ (\%) & $6(10.2)$ \\
\hline \multicolumn{2}{|l|}{ LVEF (\%) } \\
\hline Low (LVEF＜50) & $9(15.3)$ \\
\hline Normal (LVEF $\geq 50)$ & $50(84.7)$ \\
\hline \multicolumn{2}{|l|}{ RWMA grade (\%) } \\
\hline Normal & $5 \mathrm{I}(86.4)$ \\
\hline Mild hypokinesis & $5(8.5)$ \\
\hline Severe hypokinesis & 0 \\
\hline Akinesis & $3(5.1)$ \\
\hline Dyskinesis & 0 \\
\hline
\end{tabular}

Data as $n$ (\%) or median \pm SEM (range). TBI: Traumatic brain injury; LVEF: Left ventricular ejection fraction; RWMA: Regional wall motion abnormalities; SEM: Standard error of the mean; MERA: Morphologic end-repolarization abnormalities; ECG: Electrocardiographic; HR: Heart rate; LVH: Left ventricular hypertrophy

Table 3: Association between ECG finding and cardiac dysfunction in $\mathbf{5 9}$ patients with isolated TBI

\begin{tabular}{|c|c|c|}
\hline & OR $(95 \% \mathrm{Cl})$ & $P$ value \\
\hline $\mathrm{HR}>100$ bpm $(n=13 ; 22 \%)$ & $4.17(1.02-17.05)$ & 0.04 \\
\hline Arrhythmia $(n=12 ; 20.3 \%)$ & $2.86(0.67-12.11)$ & 0.14 \\
\hline Prolong PR interval $(n=4 ; 6.8 \%)$ & $1.25(1.10-1.43)$ & 0.32 \\
\hline Prolong QRS duration $(n=7 ; 11.9 \%)$ & $1.91(0.32-11.45)$ & 0.47 \\
\hline Prolong QTc $(n=25 ; 42.4 \%)$ & $9.0(1.74-46.65)$ & 0.003 \\
\hline $\operatorname{LVH}(n=6 ; 10.2 \%)$ & $1.16(0.12-11.08)$ & 0.89 \\
\hline ST depression $(n=2 ; 3.4 \%)$ & $0.21(0.12-3.70)$ & 0.25 \\
\hline ST elevation $(n=2 ; 3.4 \%)$ & $0.8 \mathrm{I}(0.7 \mathrm{I}-0.92)$ & 0.49 \\
\hline Q wave $(n=4 ; 6.8 \%)$ & $0.67(0.63-7.93)$ & 0.73 \\
\hline Inverted T wave $(n=7 ; 11.9 \%)$ & $0.52(0.87-3.14)$ & 0.47 \\
\hline $\operatorname{MERA}(n=6 ; 10.2 \%)$ & $5.63(1.96-32.94)$ & 0.03 \\
\hline
\end{tabular}

Data as $n(\%), \mathrm{OR}, 95 \% \mathrm{Cl}$ and $P$ value. LVH: Left ventricular hypertrophy; HR: Heart rate; OR: Odds ratio; Cl: Confidence interval; TBI: Traumatic brain injury; ECG: Electrocardiographic; MERA: Morphological end-repolarization. abnormalities 
brain injury, with greater ST and QT changes associated with a worsened neurologic outcome. ${ }^{[9,18]}$ In aneurysmal $\mathrm{SAH}, \mathrm{QTc}$ prolongation is common and occurs over a protracted course; in addition, dynamic ventricular arrhythmias tend to be common as well. ${ }^{[19]}$ In traumatic $\mathrm{SAH}, \mathrm{QTc}$ prolongation occurs as well, similar to nontraumatic $\mathrm{SAH}$, with greater prolongation as severity worsens. ${ }^{[18]}$

An increased understanding of the brain-heart interaction has occurred over the last several decades, as the pathophysiology has better been elucidated. Cardiac dysfunction has been described after such diverse neurologic injuries including seizures, ischemia, $\mathrm{SAH}$, and even emotional stress. ${ }^{[13,14,20]}$ Central to the pathophysiology of brain-heart interactions is a catecholamine excess state, ${ }^{[15]}$ with a possible contributing component of the inflammatory cascade, ${ }^{[21,22]}$ resulting in cardiac dysfunction, neurogenic pulmonary edema, and likely other organ injuries. ${ }^{[23,24]}$ This is further evidenced by findings on myocardial biopsy of patients with neurologic injury-induced brain death consistent with contraction band necrosis, the classic cardiac lesion of a catecholamine-excess state. ${ }^{[25]}$

Hypotension after TBI is associated with worse morbidity and mortality. ${ }^{[2,3]}$ In addition, vasopressor choice for treatment of hypotension is often empiric, with phenylephrine being the likely first choice, despite evidence to suggest the norepinephrine may improve CPP. ${ }^{[26]}$ A subset of patients with hypotension and specific ECG abnormalities may have cardiac dysfunction; these patients might benefit from alternate vasopressor support for treatment. Thus, a screening ECG in TBI patients with hypotension may provide information on which patients require a formal echocardiogram and possible modification of vasopressor therapy.

There are several limitations to our study. First, the retrospective nature of our study makes it difficult to establish cause and effect. Second, we allowed echocardiograms to be reported within $72 \mathrm{~h}$ of admission and was driven by clinical care, not study design; thus, cardiac dysfunction may have occurred earlier and may have been even more severe earlier after TBI. Furthermore, the patients who received an echocardiogram early in their care may have been sicker, thus creating a possible selection bias. Lastly, there remains residual confounding by other ICU events that may have affected the ability to attribute cardiac dysfunction to TBI, although other causes of cardiac dysfunction such as hospital-acquired sepsis tend to be later complications of critical illness.
In summary, our study suggests that repolarization abnormalities (prolonged QTc and MERA), but not ischemic-like ECG changes, are associated with abnormal echocardiographic findings in isolated TBI patients. A 12-lead ECG may be an important and inexpensive screening tool to evaluate isolated TBI patients for cardiac dysfunction prior to further diagnostic studies or interventions. Due to the inherent limitation of our retrospective study, future studies should examine TBI-induced cardiac dysfunction in a prospective fashion.

\section{References}

1. Rutland-Brown W, Langlois JA, Thomas KE, Xi YL. Incidence of traumatic brain injury in the United States, 2003. J Head Trauma Rehabil 2006;21:544-8.

2. Jeremitsky E, Omert L, Dunham CM, Protetch J, Rodriguez A. Harbingers of poor outcome the day after severe brain injury: Hypothermia, hypoxia, and hypoperfusion. J Trauma 2003;54:312-9.

3. Chesnut RM, Marshall LF, Klauber MR, Blunt BA, Baldwin N, Eisenberg HM, et al. The role of secondary brain injury in determining outcome from severe head injury. J Trauma 1993;34:216-22.

4. Zafar SN, Millham FH, Chang Y, Fikry K, Alam HB, King DR, et al. Presenting blood pressure in traumatic brain injury: A bimodal distribution of death. J Trauma 2011;71:1179-84.

5. Pietropaoli JA, Rogers FB, Shackford SR, Wald SL, Schmoker JD, Zhuang J. The deleterious effects of intraoperative hypotension on outcome in patients with severe head injuries. J Trauma 1992;33:403-7.

6. Sharma D, Brown MJ, Curry P, Noda S, Chesnut RM, Vavilala MS. Prevalence and risk factors for intraoperative hypotension during craniotomy for traumatic brain injury. J Neurosurg Anesthesiol 2012;24:178-84.

7. Samuels MA. The brain-heart connection. Circulation 2007;116:77-84.

8. Grunsfeld A, Fletcher JJ, Nathan BR. Cardiopulmonary complications of brain injury. Curr Neurol Neurosci Rep 2005;5:488-93.

9. Fan X, Du FH, Tian JP. The electrocardiographic changes in acute brain injury patients. Chin Med J (Engl) 2012;125:3430-3.

10. Junttila E, Vaara M, Koskenkari J, Ohtonen P, Karttunen A Raatikainen $\mathrm{P}$, et al. Repolarization abnormalities in patients with subarachnoid and intracerebral hemorrhage: Predisposing factors and association with outcome. Anesth Analg 2013;116:190-7.

11. Lowensohn RI, Weiss M, Hon EH. Heart-rate variability in brain-damaged adults. Lancet 1977;1:626-8.

12. Baguley IJ, Heriseanu RE, Felmingham KL, Cameron ID. Dysautonomia and heart rate variability following severe traumatic brain injury. Brain Inj 2006;20:437-44.

13. Wittstein IS, Thiemann DR, Lima JA, Baughman KL, Schulman SP, Gerstenblith G, et al. Neurohumoral features of myocardial stunning due to sudden emotional stress. N Engl J Med 2005;352:539-48.

14. Kono T, Morita H, Kuroiwa T, Onaka H, Takatsuka H, Fujiwara A. Left ventricular wall motion abnormalities in patients with subarachnoid hemorrhage: Neurogenic stunned myocardium. J Am Coll Cardiol 1994;24:636-40.

15. Hinson HE, Sheth KN. Manifestations of the hyperadrenergic state after acute brain injury. Curr Opin Crit Care 2012;18:139-45.

16. Zygun DA, Kortbeek JB, Fick GH, Laupland KB, Doig CJ. Non-neurologic organ dysfunction in severe traumatic brain injury. Crit Care Med 2005;33:654-60.

17. Coghlan LA, Hindman BJ, Bayman EO, Banki NM, Gelb AW, Todd MM, et al. Independent associations between electrocardiographic abnormalities and outcomes in patients with aneurysmal subarachnoid hemorrhage: Findings from the intraoperative hypothermia aneurysm surgery trial. Stroke 2009;40:412-8.

18. Collier BR, Miller SL, Kramer GS, Balon JA, Gonzalez LS $3^{\text {rd }}$. 
Traumatic subarachnoid hemorrhage and QTe prolongation. J Neurosurg Anesthesiol 2004;16:196-200.

19. Hravnak M, Frangiskakis JM, Crago EA, Chang Y, Tanabe M, Goresan $J 3^{\text {rd }}$, et al. Elevated cardiac troponin I and relationship to persistence of electrocardiographic and echocardiographic abnormalities after aneurysmal subarachnoid hemorrhage. Stroke 2009;40:3478-84.

20. James P, Ellis CJ, Whitlock RM, McNeil AR, Henley J, Anderson NE. Relation between troponin $\mathrm{T}$ concentration and mortality in patients presenting with an acute stroke: Observational study. BMJ 2000;320:1502-4.

21. Ako J, Sudhir K, Farouque HM, Honda Y, Fitzgerald PJ. Transient left ventricular dysfunction under severe stress: Brain-heart relationship revisited. Am J Med 2006;119:10-7.

22. Mashaly HA, Provencio JJ. Inflammation as a link between brain injury and heart damage: The model of subarachnoid hemorrhage. Cleve Clin J Med 2008;75 Suppl 2:S26-30.
23. Davison DL, Terek M, Chawla LS. Neurogenic pulmonary edema. Crit Care 2012;16:212.

24. Richard C. Stress-related cardiomyopathies. Ann Intensive Care 2011;1:39.

25. Berman M, Ali A, Ashley E, Freed D, Clarke K, Tsui S, et al. Is stress cardiomyopathy the underlying cause of ventricular dysfunction associated with brain death? J Heart Lung Transplant 2010;29:957-65.

26. Sookplung P, Siriussawakul A, Malakouti A, Sharma D, Wang J, Souter MJ, et al. Vasopressor use and effect on blood pressure after severe adult traumatic brain injury. Neurocrit Care 2011;15:46-54.

How to cite this article: Krishnamoorthy V, Prathep S, Sharma D, Gibbons E, Vavilala MS. Association between electrocardiographic findings and cardiac dysfunction in adult isolated traumatic brain injury. Indian J Crit Care Med 2014;18:570-4.

Source of Support: Nil, Conflict of Interest: None declared.

\section{Announcement}

\section{Android App}

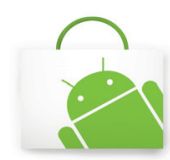

Download

Android application

A free application to browse and search the journal's content is now available for Android based mobiles and devices. The application provides "Table of Contents" of the latest issues, which are stored on the device for future offline browsing. Internet connection is required to access the back issues and search facility. The application is compatible with all the versions of Android. The application can be downloaded from https://market.android.com/details?id=comm.app.medknow. For suggestions and comments do write back to us. 